\title{
Compression of the oesophagus by the aorta $^{1}$
}

\author{
I. K. R. MCMILLAN ANDI. HYDE \\ From the Wessex Cardiac and Thoracic Centre, Southampton Chest Hospital
}

\begin{abstract}
Compression of the lower dorsal oesophagus by the aorta in elderly women sufficient to cause dysphagia has not received the attention it deserves. It is not a rare condition. The reasons for its neglect are probably two-fold: (1) the degree of dysphagia is often only slight or moderate, and (2) misdiagnosis. Occasionally, the dysphagia is serious and a threat to life. The clinical and radiological features are here described and a rational operative treatment for the severe cases is proposed. Such operative intervention does not seem to have been described previously.
\end{abstract}

With advancing age the aorta dilates, elongates, and becomes tortuous; some degree of cardiac enlargement is common, particularly involving the left ventricle; the dorsal spine tends to become kyphotic. The oesophagus, being in such close relationship to the aorta and left ventricle, is affected to a greater or lesser degree by all these changes. The resultant appearance as seen on a barium swallow is well known. That these changes of ageing can lead to dysphagia is less well known, and in fact most books and papers on this topic either deny such an association or at most state that it is rare and unimportant. Much greater importance is usually attached to the intrinsic changes in the oesophagus dus to ageing as a cause of dysphagia, namely, loss of muscle tone, reduced peristaltic activity, and a failure of the lower end to relax.

Mucklow and Smith (1954), in a paper describing the variable radiological appearances produced in the barium swallow by the aorta, noted two patients who had dysphagia. One of these was subjected to a Heller's operation and the final clinical diagnosis was 'atypical achalasia', a diagnosis with which the authors disagreed.

Keates and Magidson (1955) described seven cases of dysphagia which they believed were due to aortic compression, in some cases supplemented by enlargement of the left ventricle which effectively squeezed the lower oesophagus between these two structures. Three of these were oesophagoscoped, one showing such alarming pressure from the aorta that no attempt was made to reach the cardia. They described the different types of deflection produced by a high or low cross-over of

1Based on a paper read at the Southampton meeting of the Society of Thoracic Surgeons of Great Britain and Ireland, September 1967 the aorta behind the oesophagus and named them the 'reversed C' and 'reversed S' forms. They also noted that the crossing of the aorta behind the lower end of the oesophagus pushed the latter $\vec{\otimes}$ forwards and that the barium seemed to cascade $₫$ over this hump. There was no operative confirmation in any of these cases.

It is our contention that dysphagia from this cause is not uncommon and, whilst usually mild, ٌ it is occasionally very severe. It can be improved $\stackrel{\odot}{\mathcal{Q}}$ by treatment.

The patients are all old women, a point noted by Keates and Magidson (1955). Kyphosis, tor- $\overline{ }$ tuosity of the aorta, cardiac enlargement, and a history of heart failure are common. In a typical case the oesophagus is dilated and its lower end tapers either gradually or suddenly to a point just $\underset{\times}{\stackrel{0}{x}}$ above the diaphragm. The aorta passing behind it $\frac{0}{7}$ deflects the oesophagus forwards like a cascade 3 . which is best seen with the patient in the left $\delta$ anterior oblique position. There will be delayed $₹$ emptying but if the patient continues to drink the 의 barium a sufficient head of pressure will be built $\rightarrow$ up to force it through at an increased rate. On cessation of drinking the pressure falls and empty- $\bar{N}$ ing stops. A significant residue may remain in the $\sigma$ oeosphagus for a long time. The examination is $\tilde{N}$ best performed with the patient erect since in old $\underset{\omega}{N}$ people some dilatation and delay in the supine 0 position is commonly the result of the intrinsic 6

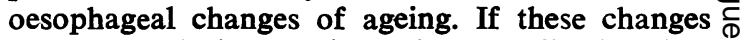
are present during gravity-assisted swallowing then $\stackrel{\mathcal{D}}{\rightarrow}$ compression can be diagnosed with greater cer- 0 tainty. Many old people show oesophageal deflec- $\bar{O}$ tion on barium swallow, but without signs of $\stackrel{\mathbb{D}}{\Omega}$ obstruction and consequently they have no symp- $\mathbb{\mathbb { D }}$ toms. In the production of obstruction and the symptoms of dysphagia the dcute angulation which 
the oesophagus is forced to take in order to reach the hiatus is the important feature. The encroachment on the oesophagus is from behind by the aorta and from in front by the anterior rim of the hiatus. The kyphosis is probably important for two reasons-it allows the oesophagus to fall backwards into the concavity of the curve, and the secondary lordosis coming out of the kyphosis forces the aorta and oesophagus forwards. The nett effect of these two forces is to increase the angulation or tightness of the curvature of the terminal portion of the oesophagus. The fact that this disorder of the oesophagus has been found only in women may be due to their greater liability to develop dorsal kyphosis. There is no obvious reason why it should be exclusive to women.

\section{ILLUSTRATIVE CASES}

CASE 1 A woman aged 85 had had recent difficulty in swallowing with fluid regurgitation and a sensation of sub-sternal sticking. A barium swallow showed the oesophagus to be dilated (Figs 1 and 2) and there was moderate hold-up. Slight reflux was elicited but no hiatus hernia. The oesophagus tapered suddenly to a point of obstruction just above the diaphragm. Oesophagoscopy was only accomplished to $35 \mathrm{~cm}$. because of the kyphosis but a bougie passed through easily. Minimal oesophagitis was present, but there was no growth and the obstruction did not feel like

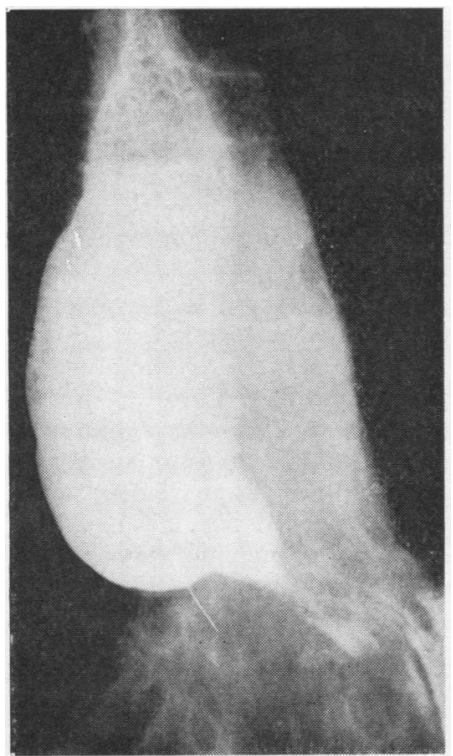

FIG. 1. Case 1. Postero-anterior view. Point of obstruction above the diaphragm. a stricture. There was no visible reflux but the cardia was not seen. The obstruction was dilated up to $29 \mathrm{~F}$.

In view of her age no further treatment was recommended and one month later swallowing was much improved. After four months the only complaint was of minimal dysphagia and the patient was discharged.

CASE 2 A woman aged 83 experienced attacks of dysphagia which frightened her but between attacks she could swallow well. In addition she suffered from chronic heart failure.

A barium swallow showed a dilated oesophagus (Fig. 3) and there was moderately severe hold-up which required a head of pressure to overcome the obstruction. Within the tight forward curvature was the calcified aorta which was also producing an impression on the gastric fundus (Fig. 4). There was no hiatus hernia or reflux.

No further investigation was undertaken on this frail patient. In the course of the next 12 months she was seen periodically by her doctor who reported that she continued to have intermittent dysphagia with sticking but the attacks were less alarming.

CASE 3 A woman aged 71 had consulted her doctor because of the recent onset of dysphagia. She was hypertensive (B.P. 250/105 mm. Hg) and had been treated for many years for mild heart failure. The barium swallow had been reported as normal but in fact it showed oesophageal deflection by the aorta above the diaphragm and a moderate degree of proximal dilatation. Her doctor requested a surgical opinion to exclude an organic lesion.

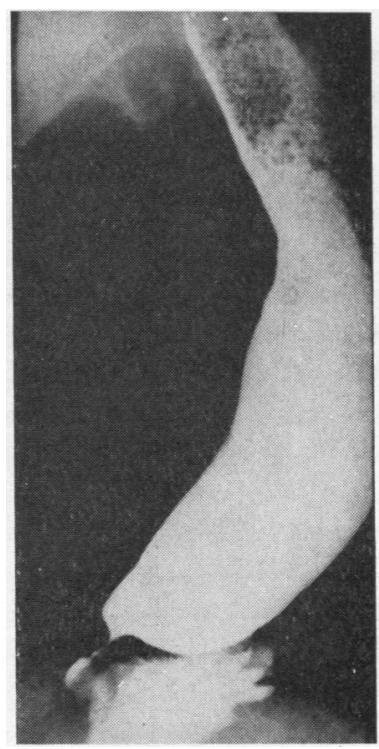

FIG. 2. Case 1. Left anterior oblique view (L.A.O.). Extreme narrowing by compression from behind. 


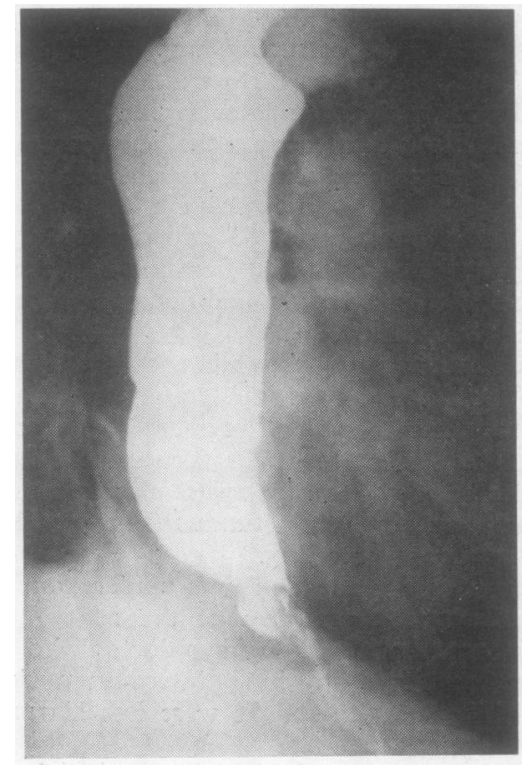

FiG. 3. Case 2. R.A.O. Obstruction above the diaphragm.

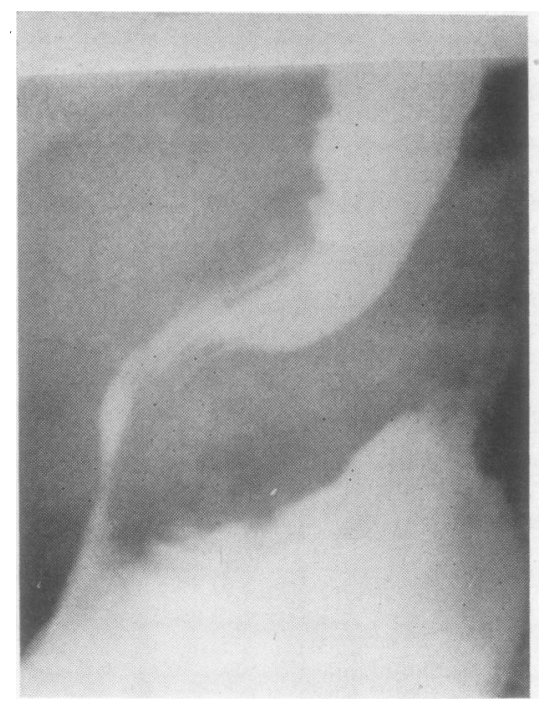

FIG. 4. Case 2. L.A.O. Characteristic forward bowing.

At oesophagoscopy the cardia was at $38 \mathrm{~cm}$., and minimal oesophagitis and minimal reflux were seen. The angle of entry was fair. There was no growth. A bougie was passed without difficulty and the obstruction dilated up to $32 \mathrm{~F}$. Operation was to be considered if no improvement resulted from the oesophagoscopy. However, two months later all dysphagia had disappeared. Treatment of the hypertension and heart failure were the only other recommendations for her future management.

CASE 4 A woman aged 78 was admitted to hospital $\frac{\overline{0}}{7}$ as an acute abdominal emergency. She complained of $\mathbb{Q}$ epigastric pain radiating upwards into the chest and jaw and had vomited bright red blood several times. There was no relevant previous history.

A barium swallow was given and the barium mixed with a resting residue of mucus and food in the oesophagus, which was slightly dilated (Fig. 5). A forward deflection was shown at its lower end and also a $\vec{x}$ striking impression over a long segment at a higher level. There was no hiatus hernia or' reflux.

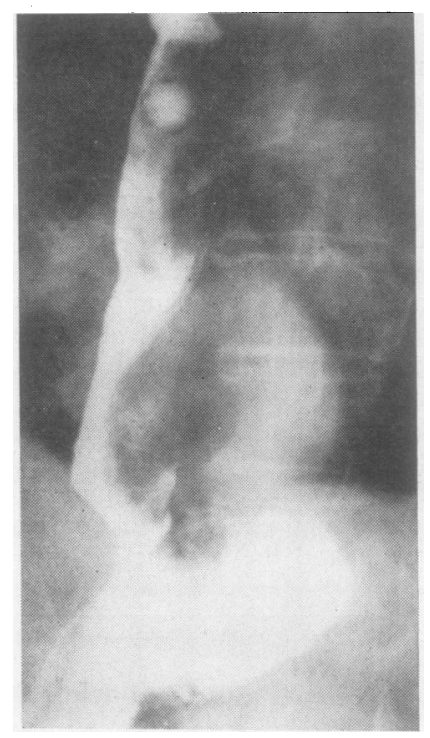

FIG. 5. Case 4. L.A.O. Food and secretions in oesophagus. Compression by the aorta on the gastric fundus and also 8 an elongated impression on the lower $7 \mathrm{~cm}$. of oesophagus.

At oesophagoscopy there was an intense oeso- 옥 phagitis and an obstruction at $35 \mathrm{~cm}$. but the latter could relax and it was therefore presumed to be muscular rather than fibrotic. There was no growth. O Dilatation up to 29F. was achieved.

A repeat of the barium swallow two days later $\stackrel{N}{0}$ showed a striking improvement and an appearance $\omega$ which would pass as normal (Fig. 6).

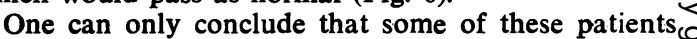
are in a delicately balanced state which is readily tilted towards further obstruction by a variety of $\stackrel{\oplus}{\oplus}$ events. A cycle of obstruction $\rightarrow$ delay $\rightarrow$ oesophagitis $\rightarrow-$ further obstruction is then initiated which has to be broken before improvement can take place.

CASE 5 A woman aged 65 complained of increasing $\stackrel{\mathbb{D}}{\stackrel{D}{D}}$ dysphagia together with pains in the epigastrium, 0 behind the sternum, and in the back. She had lost 


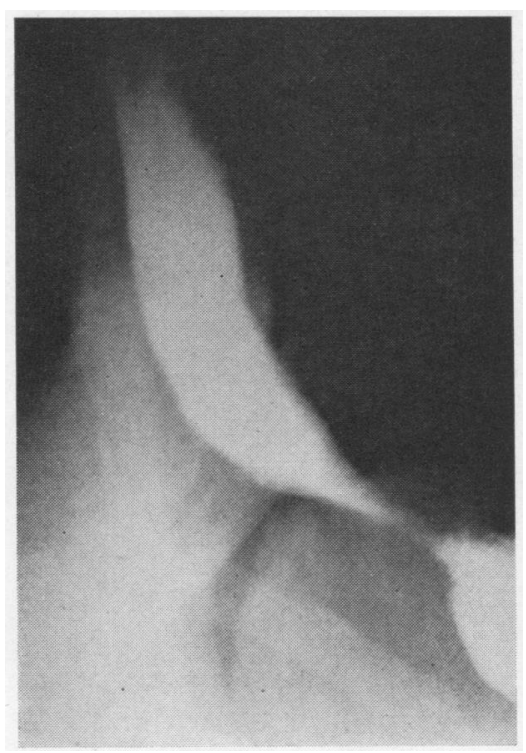

FIG. 6. Case 4. After bouginage a normal appearance except for a somewhat anterior displacement of the cardia.

$14 \mathrm{lb} .(6.4 \mathrm{~kg}$.) in weight. One year previously she had been investigated because of dysphagia but apparently nothing was found. B.P. $140 / 84 \mathrm{~mm}$. Hg.

A barium swallow showed a dilated oesophagus with delayed emptying and a gradual tapering of its lower end to a point above the diaphragm. There was a characteristic deflection at its lower end (Fig. 7). The aorta was very dilated and elongated.

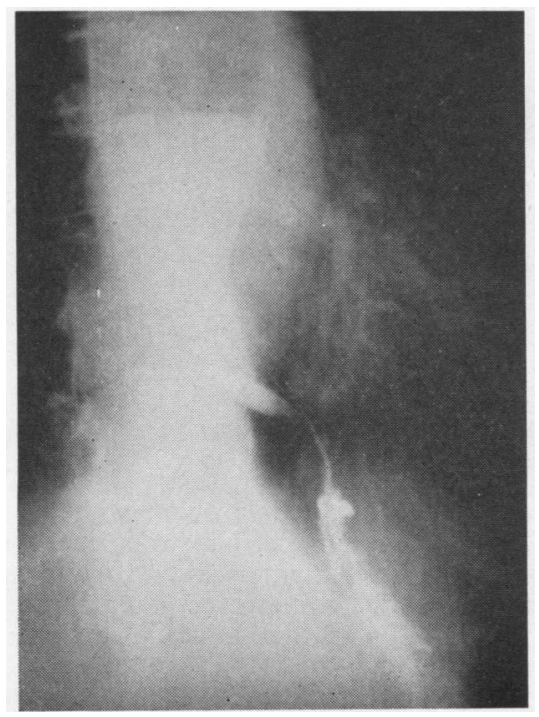

FIG. 7. Case 5. Characteristic deflection and compression.
Oesophagoscopy showed much fluid content and an oesophagitis was noted. The oesophagoscope was passed to $40 \mathrm{~cm}$. and the angle of entry into the stomach was normal.

At operation the oesophagus was found to be compressed between the left ventricle and the aorta, the latter being twice normal size. Slight muscle thickening in the oesophagus was noted. The oesophagus was transposed forwards 1 in. $(2.5 \mathrm{~cm}$.) and a Heller's muscle split was performed.

Swallowing was improved and a repeat barium examination two months later confirmed this; the lumen at the hiatus had been widened (marked by the clips in Fig. 8) but the deflection persisted and this was still causing a little hold-up. There was a normal

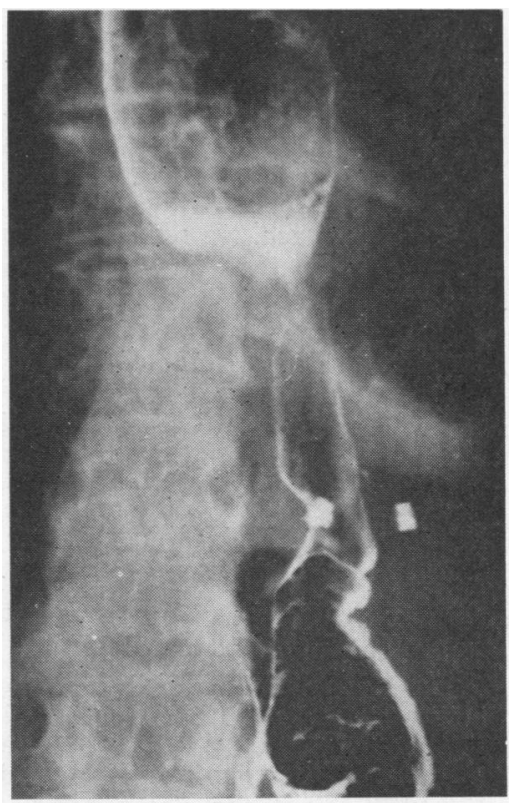

FIG. 8. Case 5. After operation. Oesophageal hiatus marked by clips. Gas in stomach. Good width of lumen. Pronounced aortic impression $7 \mathrm{~cm}$. above the cardia. Dilatation persists but the oesophagus has emptied of barium.

gas bubble in the fundus. On lying down some incompetence of the cardia was found.

The complaints of pain were unchanged and this may be explained by the unusual dilatation of the aorta.

CASE 6 A woman aged 75 complained of abdominal pain and vomiting. A diagnosis of cardiac ischaemia had been made on the basis of atrial fibrillation, heart failure, and the E.C.G. changes. However, six months later the pains and vomiting were no better and she had lost $21 \mathrm{lb}$. $(9.5 \mathrm{~kg}$.) in weight. She stated that 'life was miserable'. 


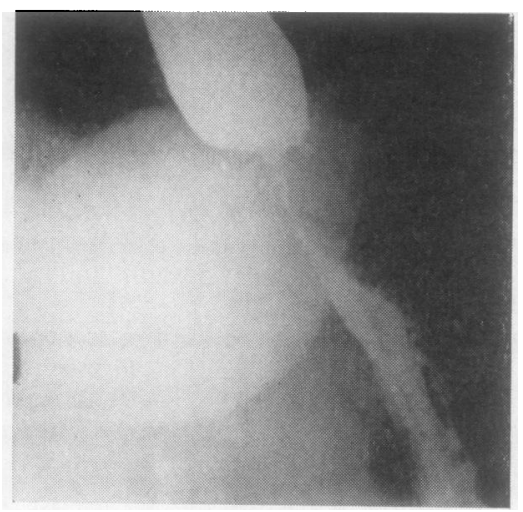

FIG. 9. Case 6. Persistent obstruction of sinister appearance.

A barium swallow showed moderate dilatation of the oesophagus with hold-up at a persistent obstruction thought to be neoplastic (Fig. 9). There was no gas bubble in the stomach. No reflux or hiatus hernia was found.

At oesophagoscopy there was no oesophagitis or growth and the obstruction which was found at 35 $\mathrm{cm}$. seemed to be due to extrinsic compression rather than a stricture. It was dilated to $32 \mathrm{~F}$.

There was no improvement and one week later she was totally obstructed. A repeat oesophagoscopy showed an intense oesophagitis and the obstruction was dilated with difficulty. A biopsy showed no tumour.

At operation a thoraco-abdominal approach was made in the belief that this was a neoplasm of the gastric fundus. There was no neoplasm and internal exploration through a gastrotomy revealed no evidence of a stricture of the oesophagus. The normal oesophagus was being compressed by the aorta and left ventricle. It was transposed forwards and laterally 1 in. $(2.5 \mathrm{~cm}$.), the crura being closed behind and the oesophagus fixed to the tendinous diaphragm by circumferential stitches.

A repeat barium swallow showed the lumen at the lower end to be of good calibre and there was no hold-up. The deflection was still present.

One year later the most that she could be persuaded to admit to was an occasional sensation of sticking at the supra-sternal notch. She had noticed a reduction in sputum volume. At this time another barium swallow (Fig. 10) showed very rapid emptying of the oesophagus. It was not possible to produce any 'buildup' of barium no matter how quickly she swallowed. Within 1 second of the completion of such rapid swallowing the oesophagus was quite empty, which is unusual even in normal old people. There was no reflux or hiatus hernia.

CASE 7 A woman aged 63 had a very severe kyphosis due to old tuberculous disease of the dorsal spine and, in addition. severe rheumatoid arthritis of both

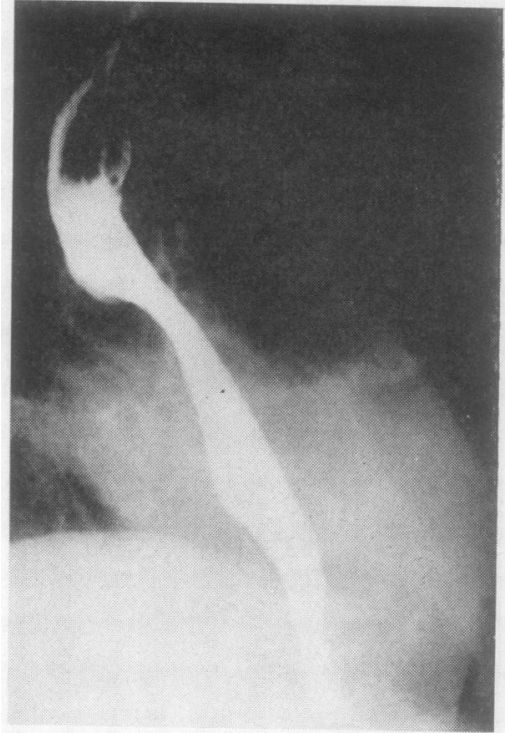

FIG. 10. Case 6. After operation. Good width of lumen but deflection persists.

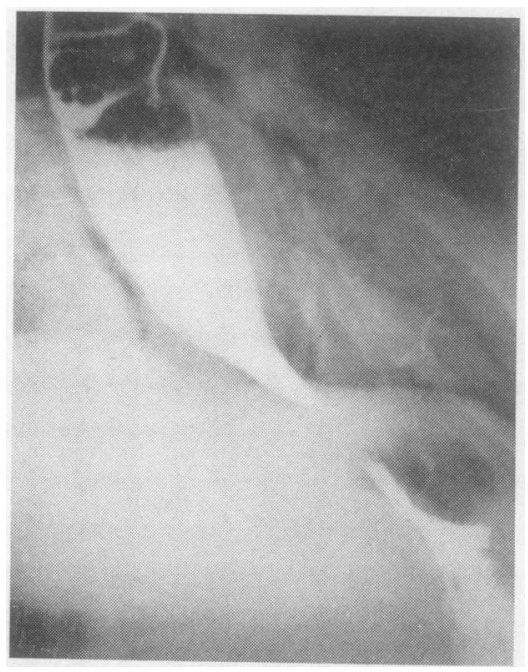

FIG. 11. Case 7. Gradual taper. Small gas bubble in stomach.

hips. She could only hobble about with the help of sticks. For three years she had experienced progressive dysphagia. A barium swallow showed the oesophagus to be dilated and tapered gradually to a point. There was hold-up of the barium. The stomach contained a gas bubble (Fig. 11).

No further investigation was attempted at this time but four months later her condition was deteriorating seriously. She was anaemic and had lost weight. 
Epigastric and retro-sternal pain was complained of and food stuck unless it was finely chewed. Even rapid drinking produced sticking.

Oesophagoscopy was accomplished to $25 \mathrm{~cm}$. only and a tube could not be passed into the stomach. At operation the oesophagus was normal but tightly clamped between the aorta and left ventricle. It was transposed forwards and laterally 1 in. $(2.5 \mathrm{~cm}$.).

Post-operatively she developed dilatation of the stomach and duodenum (the so-called duodenomesenteric ileus) which responded to conservative measures. There was no further dysphagia and a repeat barium swallow showed that there was no dilatation or delay (Fig. 12). There was a good lumen

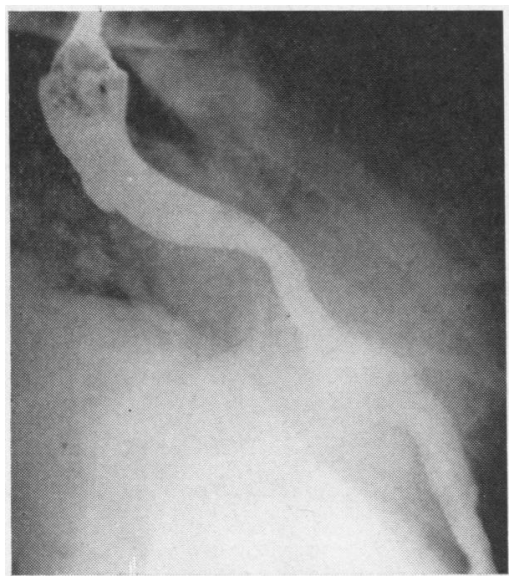

FIG. 12. Case 7. After operation. Dilatation no longer present. Good lumen width. Larger gas bubble in stomach.

width at the lower end but some deflection higher up was still present, as is to be expected. The extreme kyphosis no doubt explains the earlier onset of symptoms in this case. Seven months later there was no dysphagia but free eructation and some nausea were present. The patient was well satisfied with the result.

\section{DISCUSSION}

The first four cases described are examples of aortic compression but proof is not available. In the last two years we have seen 10 cases which satisfy for us the criteria for this diagnosis. They come from a general radiological practice as well as from a thoracic surgical unit, and this explains the high incidence of the severe types. We believe there are many other cases in our area of which we are unaware.

The last three cases were operated on and proof of severe compression was obtained. This compression was the most important element in the production of their dysphagia but it is conceded that in case 5 the muscle thickening at the lower end of the oesophagus may have been contributory. However, this case was treated many years ago (not included in the 10 mentioned above) and our present attitude would be to omit the muscle split as being irrelevant. It is also probable that the pain which was such a feature of this case was due to the aneurysmal enlargement of the aorta.

From the radiological point of view there are four parts to this diagnosis:

(1) Oesophageal deflection of characteristic type.

(2) Significant hold-up. By this is meant more than the usual small residue which is commonly found in old age.

(3) Dilatation-but again with the understanding that some dilatation is not uncommon in old age. However, this 'normal' dilatation of old age is transient.

(4) A persistently narrowed segment.

Difficulties in diagnosis are likely to arise if one or more of these features is absent or inconspicuous. For instance, if the tight forward curvature of the lower end of the oesophagus is not apparent then a mistaken diagnosis of achalasia is likely to be made. Points against this would be the presence of a gas bubble in the stomach, absence of response to octyl nitrite, and the different behaviour to a 'head of pressure'. The left anterior oblique projection is the best position to show the forward bowing of the lower end of the oesophagus. A peptic stricture may be simulated, but in our cases there was no hiatus hernia and only one showed slight gastro-oesophageal reflux. A neoplastic stricture, particularly from a carcinoma arising in the gastric fundus or from metastases encircling the cardia, is another difficult problem in differentiation, especially as the narrowed segment may not be accessible to the oesophagoscope because of the kyphosis and then exploration may be necessary to make sure, as in case 6.

Oesophagoscopy is an essential part of the investigation in order to eliminate any other pathological lesion but it may not be diagnostic. It may show appearances suspicious of extrinsic compression and this, together with the radiological features, makes the diagnosis a likely one. In mild cases it seems to have therapeutic value since, to our surprise, three patients improved after this alone.

Antero-lateral transposition of the oesophagus is the surgical method of choice as it is simple to do and at operation the anatomical arrangement causing the dysphagia is easily identified. 
After a successful operation the deflection of the oesophagus by the aorta can be expected to persist and there may indeed be some residual dilatation but the crucial tests are the transient nature of the dilatation, the absence of hold-up, and rapid emptying.

The following classification indicates the range of these cases:

(1) Oesophageal deflection only, an incidental finding without symptoms.

(2) Mild dysphagia. Dilatation and hold-up will be present but the symptoms are so inconspicuous that often no further action is taken. The barium swallow may have excluded other causes with reasonable certainty and further investigation may not be considered justified because of age or infirmity. The patient is likely to suffer intermittent, mild symptoms for the rest of her life.

(3) Moderately severe dysphagia. Oesophagoscopy and/or bouginage will usually cause some improvement which may last the rest of the patient's life, but it may have to be repeated, in which case the patient is entering the fourth group.
(4) Severe dysphagia with a deteriorating clinical state. Bouginage may not produce any improve- $\bar{C}$ ment or any such improvement may be short-lived. The patient may require operation.

Any procedure which frees the oesophagus from the hiatus may suffice, and a Heller's opera- is tion performed in the mistaken belief that achal- $\vec{\circ}$ asia is the cause of the symptoms may improve the condition. However, a more rational approach $\vec{\omega}$ is to move the oesophagus forwards and laterally away from the obstruction. This will give con- $\vec{x}$ siderable if not complete relief. In transposing the oesophagus care must be taken to maintain its correct anatomical position so that the cardia is 1 in. $(2.5 \mathrm{~cm}$.) below the diaphragm. The end result should be an oesophagus with no obstruction, no hiatus hernia, and no reflux.

\section{REFERENCES}

Keates, P. G., and Magidson, O. (1955). Dysphagia associated with sclerosis of the aorta. Brit. J. Radiol., 28, 184.

Mucklow, E. H., and Smith, O. E. (1954). Dysphagia and unusual radiographic appearances associated with the variable relation ships of the aorta and lower oesophagus. J. Fac. Radiol. (Lond.), 6,88. 